\title{
Higher Order Differential Attack on Step-Reduced Variants of Luffa v1
}

\author{
Dai Watanabe ${ }^{1}$, Yasuo Hatano ${ }^{1}$, Tsuyoshi Yamada ${ }^{2}$, and Toshinobu Kaneko ${ }^{2}$ \\ 1 Systems Development Laboratory, Hitachi, Ltd., \\ 292 Yoshida-cho, Totsuka-ku, Yokohama, 244-0817, Japan \\ 2 Science University of Tokyo, \\ 2641 Yamazaki, Noda, Chiba, 278-8510, Japan \\ dai. watanabe.td@hitachi.com
}

\begin{abstract}
In this paper, a higher order differential attack on the hash function Luffa $\mathrm{v} 1$ is discussed. We confirmed that the algebraic degree of the permutation $Q_{j}$ which is an important non-linear component of Luffa grows slower than an ideal case both by the theoretical and the experimental approaches. According to our estimate, we can construct a distinguisher for step-reduced variants of Luffa v1 up to 7 out of 8 steps by using a block message. The attack for 7 steps requires $2^{216}$ messages. As far as we know, this is the first report which investigates the algebraic property of Luffa v1. Besides, this attack does not pose any threat to the security of the full-step of Luffa v1 nor Luffa v2.
\end{abstract}

Keywords: Hash function, Luffa, Higher order differential attack, Nonrandomness.

\section{Introduction}

A cryptographic hash function has a lot of application such as a digital signature and a message authentication code. Recently, several important breakthroughs have been made in the cryptanalysis against hash functions and they imply that most of the currently used standard hash functions are vulnerable against new attacks. In these circumstances, National Institute of Standards and Technology (NIST) decided to organize Cryptographic Hash Algorithm Competition (The SHA-3 competition) [13, and started to call for algorithms.

Luffa [6] is a family of hash functions submitted to the SHA-3 competition and was selected as one of the second round candidates. Luffa modified its algorithm at the beginning of the second round and the current algorithm is called Luffa v2. Throughout this document, we discuss the algorithm submitted to Round 1 (Luffa v1) and denote it Luffa. The self-security evaluations in the supporting document for the Round 1 [7] mainly discuss generic attacks and differential cryptanalysis. Besides, analyses based on algebraic approach is not discussed seriously in the document. In this paper, we are going to investigate the algebraic property of step-reduced variants of Luffa by a higher order differential attack. 
An application of a higher order difference to cryptanalysis was suggested by Lai [1] and Knudsen firstly presented the higher order differential attack to a block cipher 10. The higher order differential attack is a tool to analyze the algebraic property of the target function, especially its algebraic degree. The application to stream ciphers was proposed by Dinur and Shamir 9] and Aumasson et al. proposed a cube tester [12] which intends to detect the non-randomness of the target function. The cube tester has been applied not only to stream ciphers, but also to several hash functions submitted to the SHA-3 competition such as MD6 and Hamsi. Recently, Aumasson and Meier proposed the zero-sum attack which is an application of the higher order differential attack [3].

In this paper, firstly we confirm that the algebraic degree of $Q_{j}$ grows slower than an ideal case both by the theoretical estimate and the experiments. According to our estimate, we can construct a distinguisher for reduced step Luffa up to 7 out of 8 steps by using a block message. The attack for 7 steps requires $2^{216}$ messages. As far as we know, this is the first report which investigates the algebraic property of Luffa v1. Besides, this attack does not pose any threat to the security of the full-step of Luffa v1 nor Luffa v2.

The rest of this paper is organized as follows: Firstly the specification of Luffa is briefly introduced in Section 2, Secondly the definition of the higher order difference and its basic property is introduced in Section 3. The increase of the algebraic degree by the iteration of the step function is investigated in Section 4. Then the higher order differential attack on step-reduced variant of the permutation $Q_{j}$ and its extension to the hash function is given in Section 5. We conclude the discussion in Section 6 .

\section{Specification of Luffa}

In this section, we introduce a part of the specification of Luffa which is needed to describe the attack. Please refer to [6] for the detail of the specification.

\subsection{Chaining}

The chaining of Luffa is a variant of a sponge function 45]. Figure 1 shows the basic structure of the chaining. The chaining of a hash function consists of iterations of a round function. The message is padded by $10 \ldots 0$ in order to the padded message length is divisible by 256 .

Round Function. The round function is a composition of a message injection function $M I$ and $w$ permutations $Q_{j}$ of 256 bits input (See Figure 1). Let the input of the $i$-th round be $\left(H_{0}^{(i-1)}, \ldots, H_{w-1}^{(i-1)}\right)$, then the output of the $i$-th round is given by

$$
\begin{aligned}
H_{j}^{(i)} & =Q_{j}\left(X_{j}\right), \quad 0 \leq j<w \\
X_{0}\|\cdots\| X_{w-1} & =M I\left(H_{0}^{(i-1)}, \ldots, H_{w-1}^{(i-1)}, M^{(i)}\right),
\end{aligned}
$$

where $H_{j}^{(0)}=V_{j}$. 


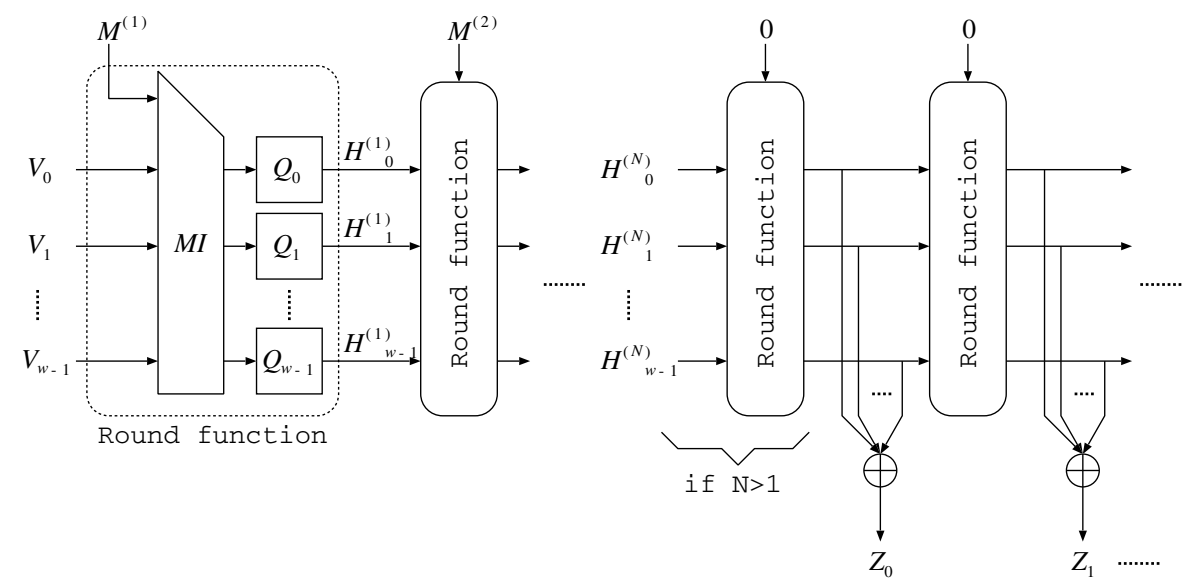

Fig. 1. The Luffa construction

In the specification of Luffa, the input length of the sub-permutation $Q_{j}$ is fixed to $n_{b}=256 \mathrm{bits}$, and the number of the sub-permutations $w$ is 3,4 and 5 for the hash length 256, 384 and 512 bits respectively.

The message injection functions can be represented by a matrix over the ring $\operatorname{GF}\left(2^{8}\right)^{32}$. The map from an 8 words value $\left(a_{0}, \ldots, a_{7}\right)$ to an element of the ring is defined by $\left(\sum_{0 \leq k<8} a_{k, l} x^{k}\right)_{0 \leq l<32}$. Note that the least significant word $a_{7}$ is the coefficient of the heading term $x^{7}$ in the polynomial representation.

Finalization. The finalization consists of iterations of an output function $O F$ and a round function with a fixed message $0 \times 00 \ldots 0$. If the number of (padded) message blocks is more than one, a blank round with a fixed message block $0 \times 00 \ldots 0$ is applied at the beginning of the finalization.

The output function $O F$ XORs all block values and outputs the resultant 256bit value. Let the output at the $i$-th iteration be $Z_{i}$, then the output function is defined by

$$
Z_{i}=\bigoplus_{j=0}^{w-1} H_{j}^{\left(N+i^{\prime}\right)}
$$

where $i^{\prime}=i$ if $N=1$ and $i^{\prime}=i+1$ otherwise. If the hash length is 256-bit, the output is $Z_{i}$. For longer hash lengths, more than one round outputs are used to generate the hash values.

\subsection{Non-linear Permutation}

The permutation $Q_{j}$ is defined as a composition of an input tweak and iterations of a step function Step. The number of iterations of a step function is 8 and the tweak is applied only once per a permutation. 


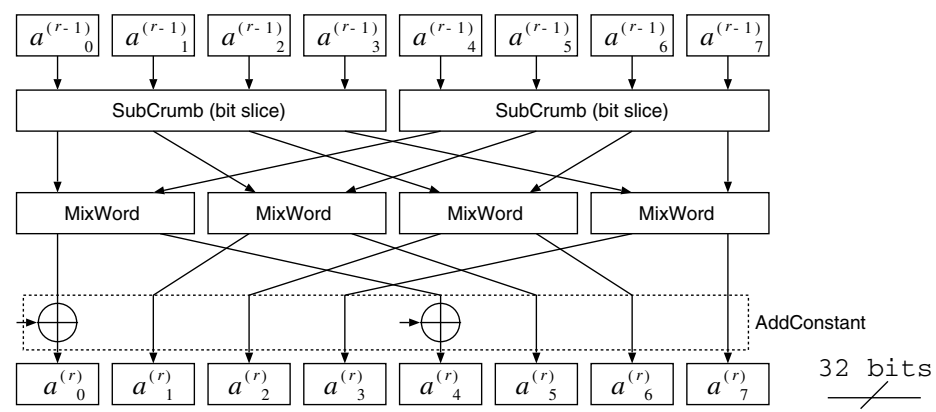

Fig. 2. The step function

At the beginning of the step function process, the 256 bits data stored in 8 32-bit registers is denoted by $a_{k}^{(r)}$ for $0 \leq k<8$. The data before applying the permutation $Q_{j}$ is denoted by $b_{k}$ and the data after the tweak is denoted by $a_{k}^{(0)}$. The step function consists of the following three functions; SubCrumb, MixWord, AddConstant. The pseudo code for $Q_{j}$ is given by

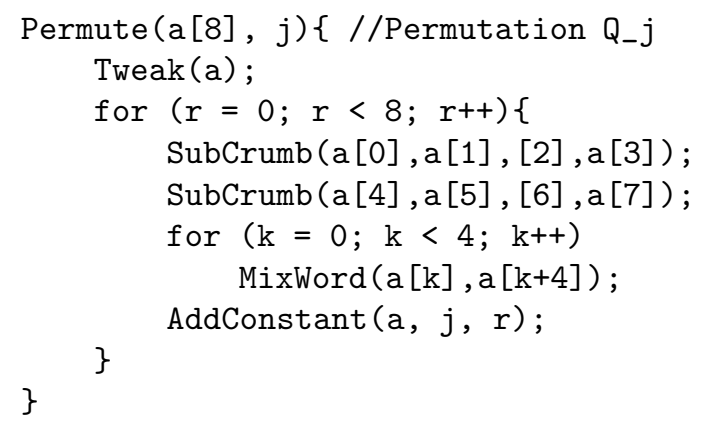

Each function is described below in turn and the tweaks are described in Section 2.2. We omit the description of AddConstant because it is not needed in this paper.

Substitution. SubCrumb substitutes $l$-th bits of $a_{0}, a_{1}, a_{2}, a_{3}$ (or $a_{4}, a_{5}, a_{6}, a_{7}$ ) by an Sbox $S$ defined by

$$
S[16]=\{7,13,11,10,12,4,8,3,5,15,6,0,9,1,2,14\} .
$$

Let the output of SubCrumb be $x_{0}, x_{1}, x_{2}, x_{3}$ ( or $x_{4}, x_{5}, x_{6}, x_{7}$ ). Then the substitution by SubCrumb is given by

$$
\begin{array}{ll}
x_{3, l}\left\|x_{2, l}\right\| x_{1, l} \| x_{0, l}=S\left[a_{3, l}\left\|a_{2, l}\right\| a_{1, l} \| a_{0, l}\right], & 0 \leq l<32, \\
x_{7, l}\left\|x_{6, l}\right\| x_{5, l} \| x_{4, l}=S\left[a_{7, l}\left\|a_{6, l}\right\| a_{5, l} \| a_{4, l}\right], & 0 \leq l<32 .
\end{array}
$$


Linear Diffusion. MixWord is a linear permutation of two words. Let the output words be $y_{k}$ and $y_{k+4}$ where $0 \leq k<4$. Then MixWord is given by the following equations:

$$
\begin{aligned}
y_{k+4} & =x_{k+4} \oplus x_{k}, \\
y_{k} & =x_{k} \lll \sigma_{1}, \\
y_{k} & =y_{k} \oplus y_{k+4}, \\
y_{k+4} & =y_{k+4} \lll \sigma_{2}, \\
y_{k+4} & =y_{k+4} \oplus y_{k}, \\
y_{k} & =y_{k} \lll \sigma_{3}, \\
y_{k} & =y_{k} \oplus y_{k+4}, \\
y_{k+4} & =y_{k+4} \lll \sigma_{4} .
\end{aligned}
$$

The parameters $\sigma_{i}$ are given by $\sigma_{1}=2, \sigma_{2}=14, \sigma_{3}=10, \sigma_{4}=1$.

Tweaks. For each permutation $Q_{j}$, the least significant four words of a 256-bit input are rotated by $j$ bits to the left in 32-bit registers. Let the $j$-th block, $k$-th word input be $b_{j, k}$ and the tweaked word (namely the input to the first step function) be $a_{j, k}^{(0)}$, then the tweak is defined by

$$
\begin{aligned}
& a_{j, k, l}^{(0)}=b_{j, k, l}, \quad 0 \leq k<4, \\
& a_{j, k, l}^{(0)}=b_{j, k,(l-j \bmod 32)}, \quad 4 \leq k<8 .
\end{aligned}
$$

\section{Higher Order Differential Attack}

An application of a higher order difference to cryptanalysis was suggested by Lai 11] and Knudsen firstly presented the higher order differential attack to a block cipher [10]. The higher order differential attack is a tool to analyze the algebraic property of the target function, especially its algebraic degree.

In this section, we give a definition of the higher order difference. In addition, the meaning of the distinguishing attack on a hash function is discussed.

\subsection{Higher Order Difference}

Let $Y=f(X)$ be a function where $X \in \mathrm{GF}(2)^{n}, Y \in \mathrm{GF}(2)^{m}$. Let $\left\{A_{1}, \ldots, A_{i}\right\}$ be a set of linearly independent vectors in $\operatorname{GF}(2)^{n}$ and $V^{(i)}$ be the sub-space spanned by these vectors. The $i$-th order difference is defined by

$$
\Delta_{V^{(i)}} f(X)=\sum_{A \in V^{(i)}} f(X+A) .
$$

In the following, $\Delta^{(i)}$ denotes $\Delta_{V^{(i)}}$ if the choice of $V^{(i)}$ does not matter in the discussion. The basic fact of the higher order difference is that $\Delta^{(D+1)} f(X)=$ 0 if the algebraic degree of $f$ with respect to $X$ is $D$. Therefore the higher 
order difference is used as the tool to evaluate the algebraic degree of the target function.

In addition to the original definition of the higher order difference, we import some terms and notations from the SQUARE attack. The SQUARE attack was proposed by Daemen et al. in 1997 as the dedicated attack on the block cipher SQUARE [8].

Let a $\Lambda$-set be a set consisting of 16 states such that their values in some crumbs (4-bit inputs to an S-box) are all different (these crumbs are called active) and their values are all equal in other crumbs (called passive). The basic idea of SQUARE attack is that a permutation preserves the status active or passive. In the higher order differential attack, this observation is useful to choose $V^{(i)}$. If $V^{(i)}$ consists of active crumbs and passive crumbs, the increase of algebraic order at the first Sbox can be ignored by replacing the inputs of the Sboxes by the corresponding outputs.

\subsection{Distinguishing Attack on a Hash Function}

We will clarify what the terminology distinguisher means in this paper.

A distinguisher for a family of functions $\mathcal{F}$ and a set of all functions which maps $\{0,1\}^{m}$ to $\{0,1\}^{n}$ is defined as a program that, given a function $f$, determines if $f$ belongs to $\mathcal{F}$. Therefore, a discussion on distinguishing attack makes sense only if the target function has a parameter. Besides, the naive definition of a collision resistant hash function does not take secret key. Therefore the application of the distinguishing attack in practice is limited to keyed applications such as HMAC. Dealing the IVs as a parameter (as in the discussion of security proof) is another possible situation.

Note that the distinguisher on a hash function (family) only detects a kind of non-randomness property of the target, does not violate collision resistance, second preimage resistance, nor preimage resistance. Even though distinguishing attacks reveal only non-randomness, we believe that this can be a first step to analyze the target function.

By the definition, it is possible to calculate the higher order differences of arbitrary functions including hash functions. Let $f$ be a randomly chosen function whose input length is $n$-bit. Then the algebraic degree of $f$ is expected $n-1$ so that the event that the $i$-th order difference $\Delta^{(i)} f$ is rarely zero if $i$ is not much less than $n$. We use this property as a distinguisher and claim that the attack is successful if such events are detected.

\section{Algebraic Degree of Non-linear Permutation $Q_{j}$}

It is pointed out in [7] that the Boolean polynomial representations of the Sbox of Luffa are sparse, especially at the highest degree. The first step of the theoretical estimate is to observe how this property affects the increase of the algebraic degree throughout the iterations of the step functions. In the following, the $r$ iterations of the step function is denoted by $Q_{j}^{(r)}$. The original permutation of Luffa is given by $Q_{j}=Q_{j}^{(8)}$. 


\subsection{Boolean Representations of Sbox}

Let the inputs and outputs of the Sbox be $x_{0, l}, x_{1, l}, x_{2, l}, x_{3, l}$ and $y_{0, l}, y_{1, l}, y_{2, l}, y_{3, l}$. Then the polynomial representations of the relations between the input and output bits are given by

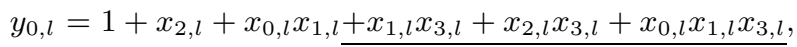

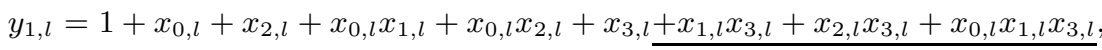

$$
\begin{aligned}
& y 2, l=1+x_{1, l}+x_{1, l} x_{3, l}+x_{2, l} x_{3, l}+x_{0, l} x_{1, l} x_{3, l}, \\
& y_{3, l}=x_{0, l}+x_{1, l}+x_{2, l}+x_{0, l} x_{1, l}+x_{1, l} x_{2, l}+x_{0, l} x_{1, l} x_{2, l}+x_{1, l} x_{3, l} .
\end{aligned}
$$

\subsection{Basic Facts}

It is clear from the simple observation of the Boolean representations of the Sbox that the terms whose degrees are more than one and which has monomial $x_{3, l}$ in $y_{0, l}, y_{1, l}, y_{2, l}$ are equal. Let $\eta_{l} \cdot x_{3, l}$ be the common part in $y_{0, l}, y_{1, l}, y_{2, l}$ and $\xi_{k, l}$ be the remainders (The strict definitions of $\eta_{l}$ and $\xi_{k, l}$ are given in Section 4.3). Then the multiplication of $y_{k, l}$ and $y_{k^{\prime}, l}$ for $k \neq k^{\prime}$ is given by

$$
y_{k, l} \cdot y_{k^{\prime}, l}=\left(\xi_{k, l}+\eta_{l} x_{3, l}\right)\left(\xi_{k^{\prime}, l}+\eta_{l} x_{3, l}\right)=\xi_{k, l} \xi_{k^{\prime}, l}+\left(\xi_{k, l}+\xi_{k^{\prime}, l}+1\right) \eta_{l} x_{3, l} .
$$

Therefore, we get $\operatorname{deg} y_{k, l} \cdot y_{k^{\prime}, l}<\operatorname{deg} y_{k, l}+\operatorname{deg} y_{k^{\prime}, l}$. This indicates that the designer's estimate of the algebraic degree of $Q_{j}^{(r)}$ is too optimistic. We should carefully estimate it.

On the other hand, MixWord() is the function which sums up $y_{k, l}$ over the subscript $l: z_{k, l}=\operatorname{MixWord}\left(y_{k}, y_{k+4}\right)_{k, l}=\sum_{\iota \in \Omega_{l}} y_{k, \iota}+\sum_{\iota \in \Omega_{l}^{\prime}} y_{k+4, \iota}$. Then $z_{k, l}$ are given by

$$
z_{k, l}=\sum_{\iota \in \Omega_{l}} \xi_{k, \iota}+\sum_{\iota \in \Omega_{l}^{\prime}} \xi_{k+4, \iota}+\sum_{\iota \in \Omega_{l}} \eta_{l} x_{3, l}+\sum_{\iota \in \Omega_{l}^{\prime}} \eta_{l}^{\prime} x_{7, l}
$$

for $k=0,1,2$, where $\eta_{l}^{\prime}$ is calculated in the same manner as $\eta_{l}$ but differs at the choice of the variables. $\eta_{l}^{\prime}$ uses $x_{4, l}, x_{5, l}, x_{6, l}, x_{7, l}$ instead of $x_{0, l}, x_{1, l}, x_{2, l}, x_{3, l}$. The property, that the higher degree terms of $y_{0, l}, y_{1, l}, y_{2, l}$ are the same, is preserved by MixWord(). AddConstant() has no influence on this property.

\subsection{Recurrence Relations about Algebraic Degree}

The observations in Section 4.2 indicates that only SubCrumb() contributes to the increase of the algebraic degree. In the following, we identify the iterations of the Sboxes (SubCrumb()) as the iterations of the step functions for the simple discussion.

Let us denote the inputs to the $l$-th Sbox in the $r$-th step function by $\left(x_{0, l}^{(r-1)}, x_{1, l}^{(r-1)}, x_{2, l}^{(r-1)}, x_{3, l}^{(r-1)}\right)$ and denote $\eta_{l}, \xi_{k, l}$ by 
$\eta_{l}^{(r)}=\eta_{l}\left(x_{0, l}^{(r)}, x_{1, l}^{(r)}, x_{2, l}^{(r)}\right)=x_{1, l}^{(r)}+x_{2, l}^{(r)}+x_{0, l}^{(r)} x_{1, l}^{(r)}$,

$\xi_{0, l}^{(r)}=\xi_{0, l}\left(x_{0, l}^{(r)}, x_{1, l}^{(r)}, x_{2, l}^{(r)}\right)=1+x_{2, l}^{(r)}+x_{0, l}^{(r)} x_{1, l}^{(r)}$,

$\xi_{1, l}^{(r)}=\xi_{1, l}\left(x_{0, l}^{(r)}, x_{1, l}^{(r)}, x_{2, l}^{(r)}\right)=1+x_{0, l}^{(r)}+x_{2, l}^{(r)}+x_{0, l}^{(r)} x_{1, l}^{(r)}+x_{0, l}^{(r)} x_{2, l}^{(r)}$,

$\xi_{2, l}^{(r)}=\xi_{2, l}\left(x_{0, l}^{(r)}, x_{1, l}^{(r)}, x_{2, l}^{(r)}\right)=1+x_{1, l}^{(r)}$,

$\xi_{3, l}^{(r)}=\xi_{3, l}\left(x_{0, l}^{(r)}, x_{1, l}^{(r)}, x_{2, l}^{(r)}\right)=x_{0, l}^{(r)}+x_{1, l}^{(r)}+x_{2, l}^{(r)}+x_{0, l}^{(r)} x_{1, l}^{(r)}+x_{1, l}^{(r)} x_{2, l}^{(r)}+x_{0, l}^{(r)} x_{1, l}^{(r)} x_{2, l}^{(r)}$.

In other words, $\eta_{l} \cdot x_{3, l}$ denotes the common terms of the polynomial representations and $\xi_{k, l}$ denotes the different terms which do not have the variable $x_{3, l}$. In addition, we denote the terms of degree $d$ in $\eta_{l}^{(r)}, \xi_{k, l}^{(r)}$ by $\eta_{l, d}^{(r)}, \xi_{k, l, d}^{(r)}$ respectively.

Now we are going to estimate the algebraic degree of $x_{k, l}^{(r)}, \eta_{l}^{(r)}, \xi_{k, l}^{(r)}$ by the recurrence relations. We approximate the relations in order to simplify their representations and Equation 1 is applied once for each variable in the estimation. Let us denote $\delta_{l}^{(r)}=\operatorname{deg} \eta_{l}^{(r-1)}+\operatorname{deg} x_{3, l}^{(r-1)}, \epsilon_{k, k^{\prime}, l}^{(r)}=\operatorname{deg} \xi_{k, l}^{(r)}+\operatorname{deg} \xi_{k^{\prime}, l}^{(r)}$. Then we have the following relations:

$$
\begin{aligned}
& \operatorname{deg} \eta_{l}^{(r)} \sim \max \left(\epsilon_{0,1, l}^{(r-1)}, \operatorname{deg} \max \left(\xi_{0, l}^{(r-1)}, \xi_{1, l}^{(r-1)}\right)+\delta_{l}^{(r-1)}\right), \\
& \operatorname{deg} \xi_{0, l}^{(r)} \sim \operatorname{deg} \eta_{l}^{(r)}, \\
& \operatorname{deg} \xi_{1, l}^{(r)} \sim \max \left(\operatorname{deg} \xi_{1, l}^{(r-1)}, \operatorname{deg} \xi_{2, l}^{(r-1)}\right)+\max \left(\operatorname{deg} \xi_{0, l}^{(r-1)}, \delta_{l}^{(r-1)}\right), \\
& \operatorname{deg} \xi_{2, l}^{(r)} \sim \max \left(\operatorname{deg} \xi_{1, l}^{(r-1)}, \delta_{l}^{(r-1)}\right), \\
& \operatorname{deg} \xi_{3, l, 2}= \max \left(\operatorname{deg} \xi_{0, l}^{(r-1)}, \operatorname{deg} \xi_{2, l}^{(r-1)}\right)+\max \left(\operatorname{deg} \xi_{1, l}^{(r-1)}, \delta_{l}^{(r-1)}\right), \\
& \operatorname{deg} \xi_{3, l, 3} \sim \max \left(\operatorname{deg} \xi_{0, l}^{(r-1)}+\operatorname{deg} \xi_{1, l}^{(r-1)}+\operatorname{deg} \xi_{2, l}^{(r-1)},\right.\left.\max \left(\epsilon_{0,1, l}^{(r-1)}, \epsilon_{0,2, l}^{(r-1)}, \epsilon_{1,2, l}^{(r-1)}\right)+\delta_{l}^{(r-1)}\right), \\
& \operatorname{deg} x_{0, l}^{(r)} \sim \max \left(\epsilon_{0,1, l}^{(r-2)}, \delta_{l}^{(r-1)}\right), \max \left(\epsilon_{0,1, l}^{(r-2)}, \epsilon_{0,2, l}^{(r-2)}, \delta_{l}^{(r-1)}\right), \\
& \operatorname{deg} x_{1, l}^{(r)} \sim \quad{ }_{l}^{(r-1)}, \\
& \operatorname{deg} x_{2, l}^{(r)} \sim \quad \max \left(\epsilon_{0,1, l}^{(r-2)}, \epsilon_{0,2, l}^{(r-2)}, \epsilon_{1,2, l}^{(r-2)}, \operatorname{deg} x_{3, l}^{(r-2)}\right)+\delta_{l}^{(r-2)}, \\
& \operatorname{deg} x_{3, l}^{(r)} \sim \max \left(\operatorname{deg} \xi_{0, l}^{(r-2)}+\operatorname{deg} \xi_{1, l}^{(r-2)}+\operatorname{deg} \xi_{2, l}^{(r-2)},\right. \\
&\left.\quad 2 \operatorname{deg} \xi_{1, l}^{(r-2)}+\operatorname{deg} \xi_{3, l}^{(r-2)}\right) .
\end{aligned}
$$

The detailed calculations to get the relations are given in Appendix A

\subsection{Theoretical Estimate of Algebraic Degrees}

Table 1 shows the pace of increase of algebraic degrees of variables $x_{k, l}, \xi_{k, l}$, $\eta_{l}$ from the recurrent relations 2 to 11 and the initial values at $r=0,1$. The input/output length of the non-linear permutation $Q_{j}^{(r)}$ is 256 bits so that the algebraic degrees are at most 256. However we put the estimated degrees as it is, even if it is more than 256 , in order to clarify the pace of increase. 
Table 1. Pace of increase of algebraic degrees (Theoretical estimate)

\begin{tabular}{|l||r|r|r|r|r|r|r|r|r|}
\hline$r$ & $x_{0, l}^{(r)}$ & $x_{1, l}^{(r)}$ & $x_{2, l}^{(r)}$ & $x_{3, l}^{(r)}$ & $\xi_{0, l}^{(r)}$ & $\xi_{1, l}^{(r)}$ & $\xi_{2, l}^{(r)}$ & $\xi_{3, l}^{(r)}$ & $\eta_{l}^{(r)}$ \\
\hline \hline 0 & 1 & 1 & 1 & 1 & 2 & 2 & 1 & 2 & 2 \\
\hline 1 & 3 & 3 & 3 & 3 & 5 & 5 & 3 & 7 & 5 \\
\hline 2 & 8 & 8 & 8 & 7 & 13 & 13 & 8 & 18 & 13 \\
\hline 3 & 20 & 20 & 20 & 18 & 33 & 33 & 20 & 46 & 33 \\
\hline 4 & 51 & 51 & 51 & 46 & 84 & 84 & 51 & 117 & 84 \\
\hline 5 & 130 & 130 & 130 & 117 & 214 & 214 & 130 & 298 & 214 \\
\hline 6 & 331 & 331 & 331 & 298 & 545 & 545 & 331 & 759 & 545 \\
\hline 7 & 843 & 843 & 843 & 759 & 1,388 & 1,388 & 843 & 1,933 & 1,388 \\
\hline 8 & 2,147 & 2,147 & 2,147 & 1,933 & 3,535 & 3,535 & 2,147 & 4,923 & 3,535 \\
\hline
\end{tabular}

\section{Higher Order Differential Attack on Luffa}

The designers of Luffa expected the algebraic degree of the permutation $Q_{j}^{(r)}$ is given by $3^{r}$ [7]. However, as shown in the previous section, the degree increases slower than the ideal case. In addition, the high order part $\eta_{l}^{(r-1)} \cdot x_{3, l}^{(r-1)}$ of the variables $x_{k, l}^{(r)}$ are common for $k=0,1,2$. We use this property to construct a distinguisher for the permutation $Q_{j}^{(r)}$. Then we extend the attack to 7 -step Luffa.

\subsection{Theoretical Estimate}

Remind the definitions of $\xi_{k, l}$ and $\eta_{l}$ that $x_{k, l}^{(r)}=\xi_{k, l}^{(r-1)}+\eta_{l}^{(r-1)} x_{3, l}^{(r-1)}$. The high order part $\eta_{l}^{(r-1)} \cdot x_{3, l}^{(r-1)}$ of the variables $x_{k, l}^{(r)}$ are common for $k=0,1,2$, so that it can be eliminated by the addition (on the binary field) $x_{k, l}^{(r)}+x_{k^{\prime}, l}^{(r)}$. We propose to use a 32-bit value $x_{k}^{(r)}+x_{k^{\prime}}^{(r)}$ as the higher order differential distinguisher. Now it is clear that the important variables in this attack are $\xi_{0, l}^{(r-1)}, \xi_{1, l}^{(r-1)}, \xi_{2, l}^{(r-1)}$, $\operatorname{not} x_{k, l}^{(r)}$.

These observations indicate that the number of steps $r$ for which $Q_{j}^{(r)}$ can be attacked can be estimated by the maximum degree of $\xi_{k, l}^{(r-2)}$. In Table 1 $\max _{k} \operatorname{deg} \xi_{k, l}^{(5)}$ is 214 so that there is a distinguisher on $Q_{j}^{(6)}$, which calculates 214-th order difference. This distinguisher for 6 steps does not depend on the choice of the input space $V^{(i)}$.

By the careful choice of the input space $V^{(i)}$, we can extend this distinguisher to 7 steps. There are two known techniques to skip the increase of the algebraic degree by applying SubCrumb () in the first step. The first one is to choose the input space $V^{(i)}$ in which the inputs to the Sboxes are active or passive. For example, if the $V^{(i)}$ takes all values in $x_{k, l}$ for $0 \leq k<8$ and $0 \leq l<t$, we can ignore the effect of $\operatorname{SubCrumb}()$ at the first step. The second technique is to 
vary only a bit per an Sbox. This technique is applicable only if the algebraic degree of the target function is small. Let us denote $m_{1}$-th bit to $m_{2}$-th bit of the variable $x$ by $x\left[m_{1} . . m_{2}\right]$. The distinguisher for 7 steps takes $x_{k}[0 . .26]$ for all $k$ as variables. In other words, all possible values of $x_{k, l}$ for $0 \leq k<8$ and $0 \leq l<27$ appear once. This distinguisher requires $2^{216}$ messages. On the other hand, $Q_{j}^{(8)}$ is not expected to be distinguishable because $\max \operatorname{deg} \xi_{k, l}^{(6)}=545>256$.

\subsection{Experimental Inspection}

By performing experiments, we check if the theoretical estimates summarized in 1 are reliable. We applied the "a bit per an Sbox" technique which is one of the two techniques to ignore the effect of the first step, as mentioned in the previous section. We did not apply the other technique. If we did, the active Sboxes are relatively sparse, so that it would be possible to skip the SubCrumb() in the second step by choosing a good alignment of the active Sboxes. However, our purpose is not to optimize the attack, but to check if the theoretical estimates summarized in Table 1 is reliable so that this kind of "unexpected" skip is not desired. Therefore, we calculated $t$-th order differences by varying the least significant $t$ bits of the 32-bit variable $x_{0}^{(0)}$ for $1 \leq t \leq 32$. We calculated each higher order difference for 100 times by randomly generating the initial states.

The experimental results are summarized in Table 2 where the numerical values show the ratio that one of the equations $x_{0}^{(r)}=x_{1}^{(r)}, x_{0}^{(r)}=x_{2}^{(r)}, x_{1}^{(r)}=$ $x_{2}^{(r)}, x_{4}^{(r)}=x_{5}^{(r)}, x_{4}^{(r)}=x_{6}^{(r)}, x_{5}^{(r)}=x_{6}^{(r)}$ holds, where $r$ means the number of steps. In other words, the values mean the ratio of the distinguishing attack being successful.

Table 3 shows the comparison between the theoretical estimates (See Table 1) and the experimental results (See Table 2).

We calculated the algebraic degree of $Q_{j}^{(r)}$ from the experimental results by the order. Let $t$ be the lowest number such that the $t$-th order differential of $x_{k}^{(r)}$ is equal to zero with probability one. The degree of $Q_{j}^{(r)}$ is formally estimated at $t-1$. This may cause the contradictions in Table 3 such that the degree of $\xi_{k, l}^{(r-2)}$ is larger than that of $x_{k, l}^{(r-1)}$ for $r=1,2$. In other cases, the Table 3 indicates that the theoretical estimates in Table 1 are very close to the experimental results in Table[2].

\subsection{Higher Order Differential Attack on the Hash Function}

The higher order differential attack on a hash function does not violate the central three requirements for a hash function, namely collision resistance, second preimage resistance, preimage resistance. On the other hand, the distinguishing attacks are useful to check whether or not the target function has pseudo-randomness

\footnotetext{
${ }^{1}$ We append a note that the $t-1$-th order differentials are rarely constants, so that it might be better to estimate the degree of $Q_{j}^{(r)}$ by $t$.
} 
Table 2. The success rates of the distinguishing attacks on the permutation $Q_{j}^{(r)}$ (Experimental results)

\begin{tabular}{|c||c|c|c|c|c|}
\hline \multicolumn{1}{|c||}{} & \multicolumn{5}{|c|}{ Number of steps } \\
\hline Order & 1 & 2 & 3 & 4 & 5 \\
\hline \hline 1 & 1.00 & 0.39 & 0.00 & 0.00 & 0.00 \\
\hline 2 & 1.00 & 1.00 & 0.12 & 0.00 & 0.00 \\
\hline 3 & 1.00 & 1.00 & 0.56 & 0.00 & 0.00 \\
\hline 4 & 1.00 & 1.00 & 0.93 & 0.00 & 0.00 \\
\hline 5 & 1.00 & 1.00 & 1.00 & 0.00 & 0.00 \\
\hline 6 & 1.00 & 1.00 & 1.00 & 0.01 & 0.00 \\
\hline 7 & 1.00 & 1.00 & 1.00 & 0.04 & 0.00 \\
\hline 8 & 1.00 & 1.00 & 1.00 & 0.16 & 0.00 \\
\hline 9 & 1.00 & 1.00 & 1.00 & 0.45 & 0.00 \\
\hline 10 & 1.00 & 1.00 & 1.00 & 0.83 & 0.00 \\
\hline 11 & 1.00 & 1.00 & 1.00 & 0.97 & 0.00 \\
\hline 12 & 1.00 & 1.00 & 1.00 & 1.00 & 0.00 \\
\hline 13 & 1.00 & 1.00 & 1.00 & 1.00 & 0.00 \\
\hline 14 & 1.00 & 1.00 & 1.00 & 1.00 & 0.00 \\
\hline 15 & 1.00 & 1.00 & 1.00 & 1.00 & 0.00 \\
\hline 16 & 1.00 & 1.00 & 1.00 & 1.00 & 0.00 \\
\hline 17 & 1.00 & 1.00 & 1.00 & 1.00 & 0.01 \\
\hline 18 & 1.00 & 1.00 & 1.00 & 1.00 & 0.00 \\
\hline 19 & 1.00 & 1.00 & 1.00 & 1.00 & 0.00 \\
\hline 20 & 1.00 & 1.00 & 1.00 & 1.00 & 0.00 \\
\hline 21 & 1.00 & 1.00 & 1.00 & 1.00 & 0.00 \\
\hline 22 & 1.00 & 1.00 & 1.00 & 1.00 & 0.03 \\
\hline 23 & 1.00 & 1.00 & 1.00 & 1.00 & 0.04 \\
\hline 24 & 1.00 & 1.00 & 1.00 & 1.00 & 0.13 \\
\hline 25 & 1.00 & 1.00 & 1.00 & 1.00 & 0.21 \\
\hline 26 & 1.00 & 1.00 & 1.00 & 1.00 & 0.38 \\
\hline 27 & 1.00 & 1.00 & 1.00 & 1.00 & 0.46 \\
\hline 28 & 1.00 & 1.00 & 1.00 & 1.00 & 0.71 \\
\hline 29 & 1.00 & 1.00 & 1.00 & 1.00 & 0.83 \\
\hline 30 & 1.00 & 1.00 & 1.00 & 1.00 & 0.85 \\
\hline 31 & 1.00 & 1.00 & 1.00 & 1.00 & 0.93 \\
\hline 32 & 1.00 & 1.00 & 1.00 & 1.00 & 0.99 \\
\hline \multicolumn{1}{|c|}{} & & & & \\
\hline
\end{tabular}

Table 3. The summary of the algebraic degrees

\begin{tabular}{|c|l||r|r|r|r|r|r|r|r|}
\hline \multicolumn{2}{|c||}{ Number of steps } & 1 & 2 & 3 & 4 & 5 & 6 & 7 & 8 \\
\hline $\left.\begin{array}{c}\text { Algebraic degree } \\
\left(\max _{0 \leq k \leq 2, l} x_{k, l}^{(r-1)}\right)\end{array}\right)$ & Theoretical estimate & 1 & 3 & 8 & 20 & 51 & 130 & - & - \\
\cline { 2 - 10 } & Experimental result & 1 & 1 & 7 & 18 & - & - & - & - \\
\hline $\begin{array}{c}\text { Distinguisher's degree } \\
\left(\max _{0 \leq k \leq 2, l} \xi_{k, l}^{(r-2)}\right)\end{array}$ & Theoretical estimate & - & 2 & 5 & 13 & 33 & 84 & 214 & - \\
\cline { 2 - 10 } & Experimental result & - & 2 & 5 & 12 & $\geq 32$ & - & - & - \\
\hline
\end{tabular}




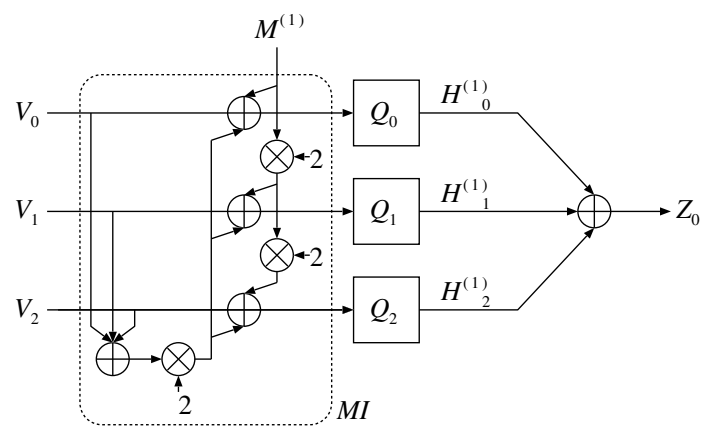

Fig. 3. Luffa for a block message $(w=3)$

which is also required to a hash function. Here we consider the higher order differential attack on the 7-step Luffa hash function.

The first point of Luffa is that there is no blank round if the message length is less than 256 bits. In this case, the message is mixed by the message injection function $M I$, permuted by non-linear permutation $Q_{j}$, then the XORed 256-bit value is output. Therefore, it might be possible to construct a distinguisher based on a higher order difference if the algebraic degree of $Q_{j}$ is smaller than 256 for all $j$. Because the only non-linear components in $L u f f a$ are $Q_{j}$ s which differ only in their tweaks and their step constants. In order to extend the distinguisher for $Q_{j}^{(7)}$ to the one for the 7-step Luffa, we consider the influences by $M I$ and the tweaks. In the following, we show that neither $M I$ nor the tweaks has influence, which is not difficult.

Firstly, the message injection function $M I$ consists of the constant multiplication over $\operatorname{GF}\left(2^{8}\right)^{32}$. This map stabilizes subspaces of $\operatorname{GF}\left(2^{8}\right)^{32}$ given by a natural injection of $\operatorname{GF}\left(2^{8}\right)^{t}$ where $t \leq 32$. Therefore there is no influence of the message injection function $M I$ if the input space is the direct product of the $\Lambda$-set. Secondly, the tweaks rotate the lower 4 words $a_{4}, a_{5}, a_{6}, a_{7}$ by $j$ bits to the left in a word. Obviously, the tweaks preserve the properties active and passive. Therefore the input space $V^{(i)}$ which cancels the influence of the message injection function also cancels that of tweaks.

These two facts indicate that the distinguisher for $Q_{j}^{(7)}$ is also applicable to the reduced step hash function as it is.

\subsection{Probabilistic Distinguisher}

Table 2 shows that the behavior of the distinguisher is probabilistic if the order is less than the expected algebraic degree. Here we discuss how to reduce the complexity of the attack.

If the target function is sufficiently random, the probability to eventually find a local collision $x_{k}=x_{k^{\prime}}$ for any $k, k^{\prime}$ is given by $6 \cdot 2^{-(32+1) / 2} \sim 2^{-14}$ and it 
is small2. Therefore $x_{k}+x_{k^{\prime}}$ can be used as a distinguisher even if the event is probabilistic. For example, Table 2 shows that 3 of 100 trials successfully found the partial collision with the 22 -th order difference for 5 steps. In this case, the computational complexity is $2^{22} \times 100 \sim 2^{28.6}$, which is smaller than the complexity of the attack with the deterministic distinguisher $2^{33}$.

On the other hand, we have no idea to theoretically estimate the frequency of this probabilistic event so that it is not clear how much the computational complexity can be reduced in the case of larger number of steps. In addition, the expected degree of the distinguisher for 8 steps is much larger than 256 so that the distinguisher is expected to include many high order terms. We expect that it is difficult to apply the higher order differential distinguisher to 8 steps if the probabilistic event can be observed more often than the deterministic event.

\subsection{Zero-Sum Attack}

Zero-sum attack was recently proposed by Aumasson and Meier [3] and it is an application of higher order differential attack. The basic idea of the zero-sum attack is to choose the $\Lambda$-set as the intermediate variables and estimate the increases of the algebraic degrees at the input and output of the target function. If the algebraic degrees (to both sides) are low, there is an certain set of inputs such that (a) their xoring is zero, and (b) the xoring of their corresponding outputs is also zero. This is a property which an ideal permutation does not have, and the zero-sum attack uses this property as the distinguisher.

By intuition the zero-sum attack enables to attack double more rounds than the original higher order differential attack. They claimed that the attack on the permutation $Q_{j}$ of $L$ uffa requires $2^{81}$ inputs. It is obvious that the number of required inputs can be reduced to $2^{33}$ due to our evaluation result (See Table 1). However, as they mentioned, it is not obvious problem to find an adequate set of messages which satisfies zero-sum property for all $Q_{j}$.

\subsection{The Higher Order Differential Attack on Luffa v2}

Luffa changed its algorithm at the beginning of the Round 2 and it is called Luffa v2. We do not describe the changes in detail, but the most significant change of Luffa v2 in terms of higher order differential attack is that a blank round in the finalization process is applied even if the message length is less than 256 bits. Therefore 16 step functions are always applied for any message block so that their algebraic degree is not likely to be less than 256 .

\section{Conclusion}

In this paper, a higher order differential attack on the hash function Luffa is discussed. We confirmed that the algebraic degree of the underlying non-linear

${ }^{2}$ In [1] Lai pointed out that $\operatorname{Prob}\left(\delta_{V_{i}} f(a)=b\right)$ is either 0 or at least $2^{i-n}$ where $f$ : $\mathrm{GF}\left(2^{\mathrm{n}}\right) \rightarrow \mathrm{GF}\left(2^{\mathrm{n}}\right)$. But this is not our case because the domain of our distinguisher is larger than the range. The probabilistic behavior of our distinguisher may be caused by the terms of high degree of $Q_{j}^{(r)}$ being sparsely distributed. 
permutation $Q_{j}$ increases slower than expected both by the theoretical estimate and the experiments. According to our estimate, we can construct a distinguisher for reduced step Luffa up to 7 out of 8 steps by using a block message. The attack for 7 steps requires $2^{216}$ messages. As far as we know, this is the first report which investigates the algebraic property of Luffa $\mathrm{v} 1$. Besides, this attack does not pose any threat to the security of the full-step of Luffa v1 nor Luffa v2.

\section{Acknowledgements}

The authors would like to thank Hirotaka Yoshida and the anonymous reviewers of FSE 2010 for their helpful comments and suggestions.

\section{References}

1. Aumasson, J.-P., Dinur, I., Meier, W., Shamir, A.: Cube Testers and Key Recovery Attacks On Reduced-Round MD6 and Trivium. In: Dunkelman, O. (ed.) FSE 2009. LNCS, vol. 5665, pp. 1-22. Springer, Heidelberg (2009)

2. Aumasson, J.-P., Dinur, I., Henzen, L., Meier, W., Shamir, A.: Efficient FPGA Implementations of High-Dimensional Cube Testers on the Stream Cipher Grain128. In: Special-purpose Hardware for Attacking Cryptographic Systems, SHARCS 2009 (2009)

3. Aumasson, J.P., Meier, W.: Zero-sum distinguishers for reduced Keccak- $f$ and for the core functions of Luffa and Hamsi (2009),

http://www.131002.net/data/papers/AM09.pdf

4. Bertoni, G., Daemen, J., Peeters, M., Van Assche, G.: Sponge Functions. In: Ecrypt Hash Workshop (2007)

5. Bertoni, G., Daemen, J., Peeters, M., Van Assche, G.: On the Indifferentiability of the Sponge Construction. In: Smart, N.P. (ed.) EUROCRYPT 2008. LNCS, vol. 4965, pp. 181-197. Springer, Heidelberg (2008)

6. De Cannière, C., Sato, H., Watanabe, D.: Hash Function Luffa: Specification. Submission to NIST SHA-3 Competition (2008),

http://www.sdl.hitachi.co.jp/crypto/luffa/

7. De Cannière, C., Sato, H., Watanabe, D.: Hash Function Luffa: Supporting Document. Submission to NIST SHA-3 Competition (2008),

http://www.sdl.hitachi.co.jp/crypto/luffa/

8. Daemen, J., Knudsen, L., Rijmen, V.: The Block Cipher Square. In: Biham, E. (ed.) FSE 1997. LNCS, vol. 1267, pp. 149-165. Springer, Heidelberg (1997)

9. Dinur, I., Shamir, A.: Cube Attacks on Tweakable Black Box Polynomials. Cryptology ePrint Archive, Report 2008/385

10. Knudsen, L.R.: Truncated and Higher Order Differentials. In: Preneel, B. (ed.) FSE 1994. LNCS, vol. 1008, pp. 196-211. Springer, Heidelberg (1995)

11. Lai, X.: Higher order derivatives and differential cryptanalysis. In: Proc. Symposium on Communication, Coding and Cryptography, pp. 227-233. Kluwer Academic Publishers, Dordrecht (1994)

12. National Institute of Standards and Technology, Secure Hash Standard (SHS), FIPS 180-2 (2002)

13. National Institute of Standards and Technology, Cryptographic hash project, http://csrc.nist.gov/groups/ST/hash/index.html 


\section{A Recurrence Relations}

The symbol " " means the simplification of the expression which (is considered) preserves the algebraic degree.

\section{A.1 Recurrence Relation of $\eta_{l}^{(r)}$}

$$
\begin{aligned}
\eta_{l}^{(r)} & =x_{1, l}^{(r)}+x_{2, l}^{(r)}+x_{0, l}^{(r)} x_{1, l}^{(r)} \\
& \sim x_{0, l}^{(r)} x_{1, l}^{(r)} \\
& =\left(\xi_{0, l}^{(r-1)}+\eta_{l}^{(r-1)} x_{3, l}^{(r-1)}\right)\left(\xi_{1, l}^{(r-1)}+\eta_{l}^{(r-1)} x_{3, l}^{(r-1)}\right) \\
& =\xi_{0, l}^{(r-1)} \xi_{1, l}^{(r-1)}+\left(\xi_{0, l}^{(r-1)}+\xi_{1, l}^{(r-1)}+1\right) \eta_{l}^{(r-1)} x_{3, l}^{(r-1)} \\
& \sim \xi_{0, l}^{(r-1)} \xi_{1, l}^{(r-1)}+\left(\xi_{0, l}^{(r-1)}+\xi_{1, l}^{(r-1)}\right) \eta_{l}^{(r-1)} x_{3, l}^{(r-1)} .
\end{aligned}
$$

\section{A.2 Recurrence Relation of $\xi_{k, l}^{(r)}$}

$$
\begin{gathered}
\xi_{0, l}^{(r)}=\xi_{0,0}^{(r)}+\xi_{0,1}^{(r)}+\xi_{0,2}^{(r)} \sim \xi_{0,2}^{(r)}=x_{0, l}^{(r)} x_{1, l}^{(r)}=\eta_{l}^{(r)} \\
\xi_{1, l}^{(r)}=\xi_{1,0}^{(r)}+\xi_{1,1}^{(r)}+\xi_{1,2}^{(r)} \\
\sim \xi_{1,2}^{(r)} \\
=x_{0, l}^{(r)} x_{1, l}^{(r)}+x_{0, l}^{(r)} x_{2, l}^{(r)} \\
=\left(\xi_{1, l}^{(r-1)}+\xi_{2, l}^{(r-1)}\right)\left(\xi_{0, l}^{(r-1)}+\eta_{l}^{(r-1)} x_{3, l}^{(r-1)}\right) \\
\xi_{2, l}^{(r)}=\xi_{2,0}^{(r)}+\xi_{2,1}^{(r)} \sim \xi_{2,1}^{(r)}=x_{1, l}^{(r)}=\xi_{1, l}^{(r-1)}+\eta_{l}^{(r-1)} x_{3, l}^{(r-1)} \\
\xi_{3, l, 2}^{(r)}=\left(x_{0, l}^{(r)}+x_{2, l}^{(r)}\right) x_{1, l}^{(r)} \\
=\left(\xi_{0, l}^{(r-1)}+\xi_{2, l}^{(r-1)}\right)\left(\xi_{1, l}^{(r-1)}+\eta_{l}^{(r-1)} x_{3, l}^{(r-1)}\right) \\
\xi_{3, l, 3}^{(r)}=x_{0, l}^{(r)} x_{1, l}^{(r)} x_{2, l}^{(r)} \\
=\left(\xi_{0, l}^{(r-1)}+\eta_{l}^{(r-1)} x_{3, l}^{(r-1)}\right)\left(\xi_{1, l}^{(r-1)}+\eta_{l}^{(r-1)} x_{3, l}^{(r-1)}\right)\left(\xi_{2, l}^{(r-1)}+\eta_{l}^{(r-1)} x_{3, l}^{(r-1)}\right) \\
\sim \xi_{0, l}^{(r-1)} \xi_{1, l}^{(r-1)} \xi_{2, l}^{(r-1)} \\
+\left(\xi_{0, l}^{(r-1)} \xi_{1, l}^{(r-1)}+\xi_{0, l}^{(r-1)} \xi_{2, l}^{(r-1)}+\xi_{1, l}^{(r-1)} \xi_{2, l}^{(r-1)}\right) \eta_{l}^{(r-1)} x_{3, l}^{(r-1)}
\end{gathered}
$$




\section{A.3 Recurrence Relation of $x_{k, l}^{(r)}$}

$$
\begin{aligned}
& x_{0, l}^{(r)}=\xi_{0,0}^{(r-1)}+\xi_{0,1}^{(r-1)}+\xi_{0,2}^{(r-1)}+\eta_{l}^{(r-1)} x_{3, l}^{(r-1)} \\
& \sim \xi_{0,2}^{(r-1)}+\eta_{l}^{(r-1)} x_{3, l}^{(r-1)} \\
& =x_{0, l}^{(r-1)} x_{1, l}^{(r-1)}+\eta_{l}^{(r-1)} x_{3, l}^{(r-1)} \\
& =\left(\xi_{0, l}^{(r-2)}+\eta_{l}^{(r-2)} x_{3, l}^{(r-2)}\right)\left(\xi_{1, l}^{(r-2)}+\eta_{l}^{(r-2)} x_{3, l}^{(r-2)}\right)+\eta_{l}^{(r-1)} x_{3, l}^{(r-1)} \\
& \sim \xi_{0, l}^{(r-2)} \xi_{1, l}^{(r-2)}+\eta_{l}^{(r-1)} x_{3, l}^{(r-1)} \text {. } \\
& x_{1, l}^{(r)}=\xi_{1,0}^{(r-1)}+\xi_{1,1}^{(r-1)}+\xi_{1,2}^{(r-1)}+\eta_{l}^{(r-1)} x_{3, l}^{(r-1)} \\
& \sim \xi_{1,2}^{(r-1)}+\eta_{l}^{(r-1)} x_{3, l}^{(r-1)} \\
& =x_{0, l}^{(r-1)} x_{1, l}^{(r-1)}+x_{0, l}^{(r-1)} x_{2, l}^{(r-1)}+\eta_{l}^{(r-1)} x_{3, l}^{(r-1)} \\
& \sim \xi_{0, l}^{(r-2)}\left(\xi_{1, l}^{(r-2)}+\xi_{2, l}^{(r-2)}\right)+\eta_{l}^{(r-1)} x_{3, l}^{(r-1)} . \\
& x_{2, l}^{(r)}=\xi_{2,0}^{(r-1)}+\xi_{2,1}^{(r-1)}+\eta_{l}^{(r-1)} x_{3, l}^{(r-1)} \sim \eta_{l}^{(r-1)} x_{3, l}^{(r-1)} . \\
& x_{3, l}^{(r)}=\xi_{3,1}^{(r-1)}+\xi_{3, l, 2}^{(r-1)}+\xi_{3, l, 3}^{(r-1)}+x_{1}^{(r-1)} x_{3, l}^{(r-1)} \\
& \sim \xi_{3, l, 3}^{(r-1)}+x_{1, l}^{(r-1)} x_{3, l}^{(r-1)} \\
& =x_{0, l}^{(r-1)} x_{1, l}^{(r-1)} x_{2, l}^{(r-1)}+x_{1, l}^{(r-1)} x_{3, l}^{(r-1)} \\
& =\left(\xi_{0, l}^{(r-2)}+\eta_{l}^{(r-2)} x_{3, l}^{(r-2)}\right)\left(\xi_{1, l}^{(r-2)}+\eta_{l}^{(r-2)} x_{3, l}^{(r-2)}\right)\left(\xi_{2, l}^{(r-2)}+\eta_{l}^{(r-2)} x_{3, l}^{(r-2)}\right) \\
& +\left(\xi_{1, l}^{(r-2)}+\eta_{l}^{(r-2)} x_{3, l}^{(r-2)}\right)\left(\xi_{3, l, 2}^{(r-2)}+\xi_{3, l, 3}^{(r-2)}+x_{1, l}^{(r-2)} x_{3, l}^{(r-2)}\right) \\
& \sim \xi_{0, l}^{(r-2)} \xi_{1, l}^{(r-2)} \xi_{2, l}^{(r-2)} \\
& +\left(\xi_{0, l}^{(r-2)} \xi_{1, l}^{(r-2)}+\xi_{0, l}^{(r-2)} \xi_{2, l}^{(r-2)}+\xi_{1, l}^{(r-2)} \xi_{2, l}^{(r-2)}+\xi_{3, l, 3}^{(r-2)}\right) \eta_{l}^{(r-2)} x_{3, l}^{(r-2)} \\
& \xi_{1, l}^{(r-2)}\left(\xi_{3, l, 3}^{(r-2)}+x_{1, l}^{(r-2)} x_{3, l}^{(r-2)}\right) .
\end{aligned}
$$

